\title{
Grafting Copolymerization of Sodium Alginate/ Acrylic Acid/ Methacrylic Acid by Gamma Radiation: Preparation and Characterization
}

\author{
H.M.M. Abo Eldahab ${ }^{a}$, R.S. Hassan ${ }^{a}$, F.A. Shehata ${ }^{a}$, S.A.El-Reefy ${ }^{a}$, S.A.Mohamed ${ }^{b}$ \\ (a) Analytical Chemistry Department, Hot Laboratories and Waste Management Center, Atomic Energy Authority, Cairo, Egypt \\ (b) Chemistry Department, Ain Shams University, Cairo, Egypt
}

\section{ARTICLE INFO}

Article history:

Received: $2^{\text {nd }}$ June 2020

Accepted: $6^{\text {th }}$ July 2020

\section{Keywords:}

Grafting copolymerization,

Gamma radiation induced,

polymerization,

Sodium alginate,

Methacrylic acid,

Swelling propert.

\begin{abstract}
Grafted copolymer (sodiumalginate (Na. Alg)- acrylic acid (AcA)methacrylic acid (MAcA)) was prepared by the gamma irradiation-induced copolymerization in the presence of $\mathrm{N}, \mathrm{N}$-methylene bis-acrylamide (MBA) as aco monomer crosslinker. The functionality, morphology and thermal stability of the prepared copolymer have been examined using FTIR spectroscopy, SEM, and TGA-DTA, respectively. The prepared material shows a smooth surface and relatively homogeneous appearance which reveals incompatibility between the monomers used. Thermal analysis of the prepared material shows stability near $400^{\circ} \mathrm{C}$. The effect of the preparation conditions on the swelling property of the prepared copolymer has been investigated and the mechanism of grafting copolymerization through the irradiation process has been also discussed. The obtained results revealed that the optimum conditions for preparing a suitable grafted copolymer were using $20 \%$ Acrylic acid, 10\% Methacrylic acid and 0.4 wt \% sodium alginate at an irradiation dose of $15 \mathrm{kGy}$ with the addition of $0.03 \%$ of MBA as a crosslinker.
\end{abstract}

\section{INTRODUCTION}

Polymers as sorbent materials have found particular importance in the preconcentration and treatment of liquid waste [1]. Since they are characterized by having ionic and complex- ability nature, polymers showed affinity towards certain ions or groups of ions from solutions. The polymers should be characterized by adaptability (non-toxicity and non-antigenicity), biodegradable and biocompatible.

The application of natural resources in the preparation of polymers showed considerable advantages since they possess the potential for substitution of chemical materials [2]. The most common natural materials used in the preparation of polymers are gelatin, albumin, chitosan and sodium alginate[3]. As one of the natural materials, sodium alginate $(\mathrm{NaAlg})$ is a linear polymer water-soluble salt of alginic acid. It is a naturally occurring heteropolysaccharide, extracted from natural brown algae [4]. Sodium alginate can be easily modified by different chemical or physical methods such as grafting copolymerization with other hydrophilic monomers such as acrylic acid [5].

The radiation-induced grafting copolymerization (RIGC) is one of the most acceptable methods for the preparation of polymeric materials of a wide range of properties. In this method, acceptable properties of the grafted copolymer can be obtained by choosing the appropriate pair or more of monomers and by controlling the conditions of the grafting process [6]. It is known that the grafting process by high energy radiation such as $\gamma$-rays improves the properties of polymeric materials [78]. In this concern, when the radiation from a $\gamma$-rays source interacts with a polymer, its energy absorbed by the polymeric material and active sites such as free radicals are produced, which in turn initiates the occurrence of different chemical reactions to occur [9-10]. 
The aim of the present work is the preparation and characterization of a novel copolymer based on using sodium alginate as a natural monomer and RIGC technique. The prepared grafted copolymer is characterized by FTIR, SEM, TGA to investigate its molecular structure, morphology, and thermal stability. Some other selected properties of the prepared grafted copolymer will be investigated and discussed to elucidate the possibility of its further use as a sorbent material.

\section{MATERIAL AND METHODS}

\subsection{Materials}

Sodium alginate ( $\mathrm{Na}$. Alg) was purchased as an alginic acid sodium salt (Fluka Chemie). Acrylic acid (AcA) and methacrylic acid (MAcA) were purchased from Sigma-Aldrich. The dissolution process was performed using de-oxygenated double distilled water which was bubbled by purging pure nitrogen gas at $80^{\circ} \mathrm{C}$.

\subsection{Preparation of Grafting Copolymer}

A general procedure for grafting copolymerization of Acrylic acid (AcA) and methacrylicacid (MAcA) onto sodium alginate ( $\mathrm{Na} \mathrm{Alg}$ ) backbone with methylene bisacrylamide (MBA) as acrosslinker using gamma irradiation technique was conducted as follows, unless otherwise stated:0.4 wt \% sodium alginate was dissolved in degassed, distilled water, following this, 20 mass $\%$ (AcA) and 10 mass $\%$ (MAcA) were added to the total solution. 0.03 mass $\% \mathrm{MBA}$ as a crosslinker was added to the reaction mixture then shaken overnight in a glass tube, after that it was subjected to Co-60 gammarays irradiation $(15 \mathrm{kGy})$. The irradiated product (grafted copolymer) was cut into small pieces and washed by acetone for removal of excess of unreacted monomers[11], washed with double distilled water and dried in an oven at $80{ }^{\circ} \mathrm{C}$ and weighed. It is worthy to mention that in case of using some other conditions, no complete formation of the uniform polymer was obtained.

\subsection{Gamma Irradiation}

The irradiation process was carried out using the Co-60 gamma cell. A cell of type MC-20 (Russia) was used at the Cyclotron Project, Inshas site, Egypt. The monomers were exposed to different doses of gamma radiation (5-30)kGy. Gamma radiation leads to the formation of free radicals, so it acts as an initiator for the polymerization process. These free radicals are active sites that crosslinked with other monomers to get the grafted copolymer.

\subsection{Characterization of the PreparedGrafted Co- polymer}

\subsubsection{Swelling studies}

The swelling property was studied to explain the behavior of the prepared grafted copolymer in water. Swelling was carried out on all samples according to ASTM D4318, 2010 and ASTM D4546, 2008 standards, respectively. Accordingly, the dried grafted copolymer was immersed in distilled water until it swelled to equilibrium. In this respect, a known dry weight $\left(\mathrm{W}_{\mathrm{i}}\right)$ of the grafted copolymer (50 $\mathrm{mg}$ ) was immersed in a known volume of distilled water $(10 \mathrm{ml})$ for 24 hours at room temperature. Swollen gels were removed from the water, dried with filter paper, and weighed $\left(\mathrm{W}_{\mathrm{f}}\right)$. The swelling percentage $(\% \mathrm{~S})$ of the gel was calculated from the following equation[12].

$$
\% S=\frac{(W f-W i)}{W i} 100
$$

Where $\mathrm{W}_{\mathrm{i}}$ is the weight of the polymer in the dried state and $\mathrm{W}_{\mathrm{f}}$ is the weight of the swollen polymer.

\subsubsection{FTIR Spectroscopic analysis}

FTIR (Fourier transformed infrared) spectrum of the copolymer was recorded by the LARA213 FT-IR spectrometer using $\mathrm{KBr}$ salt to form a disk and performed on a computerized spectrophotometer in the range of $4000-500 \mathrm{~cm}^{-1}$.

\subsubsection{Thermal analysis}

TGA and DTA measurements were carried out using a Shimadzu thermogravimetric analyzer model TGA-50 (Tokyo, Japan). The thermal stability was investigated at a heating rate of $10^{\circ} \mathrm{C} / \mathrm{min}$, under nitrogen atmosphere $(20 \mathrm{ml} / \mathrm{min})$ from room temperature up to $600^{\circ} \mathrm{C}$.

\subsubsection{Scanning Electron Microscope (SEM)}

The surface morphology of the prepared polymer was examined by JEOL-JSM 6510 LA (Japan) and used for investigation of the pore structure at high magnification of an electron beam.

\section{RESULTS AND DISCUSSION}

\subsection{Predicted Mechanism for Polymerization Process}

Sodium alginate is known to contain a large number of free hydroxyl $(\mathrm{OH})$ and carboxyl $(\mathrm{COOH})$ groups along its polymer backbones which enables it to be easily modified [13]. Meanwhile acrylic acid and 
methacrylic acid monomers have one active double bond and carboxyl hydrophilic groups that are easily binding with hydroxyl groups of water molecules to form hydrogen bonds. Accordingly, when acrylic acid and methacrylic acids are irradiated in the presence of $\mathrm{NaAlg}$ solution and MBA (crosslinker), the reaction between monomers occurs. It means that acrylic acid and methacrylic acid are anionic weak electrolytes, and have carboxylic acid groups which could develop different intermolecular interactions including electrostatic interaction, hydrogen bonds, and dipole-ion with other materials. Possible mechanism reactions for the graft polymerization of $\mathrm{Na} \mathrm{Alg} / \mathrm{AcA} / \mathrm{MAcA}$ in the presence of an MBA can be predicted [14] as shown in Scheme (1)

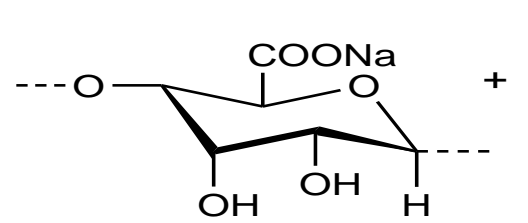

sod. alginate<smiles>C=C(C)C(=O)O</smiles>

acrylic acid

methacrylic acid

\section{Gamma ray}

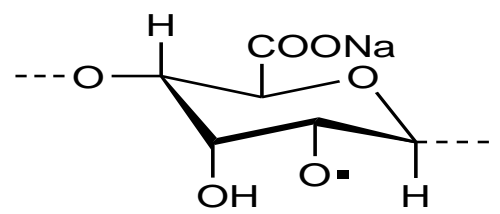<smiles>C=CC(=O)[O-]</smiles><smiles>C=C(C)C(=O)[O-]</smiles><smiles>C=CC(=O)NCNC(=O)C=C</smiles>

MBA
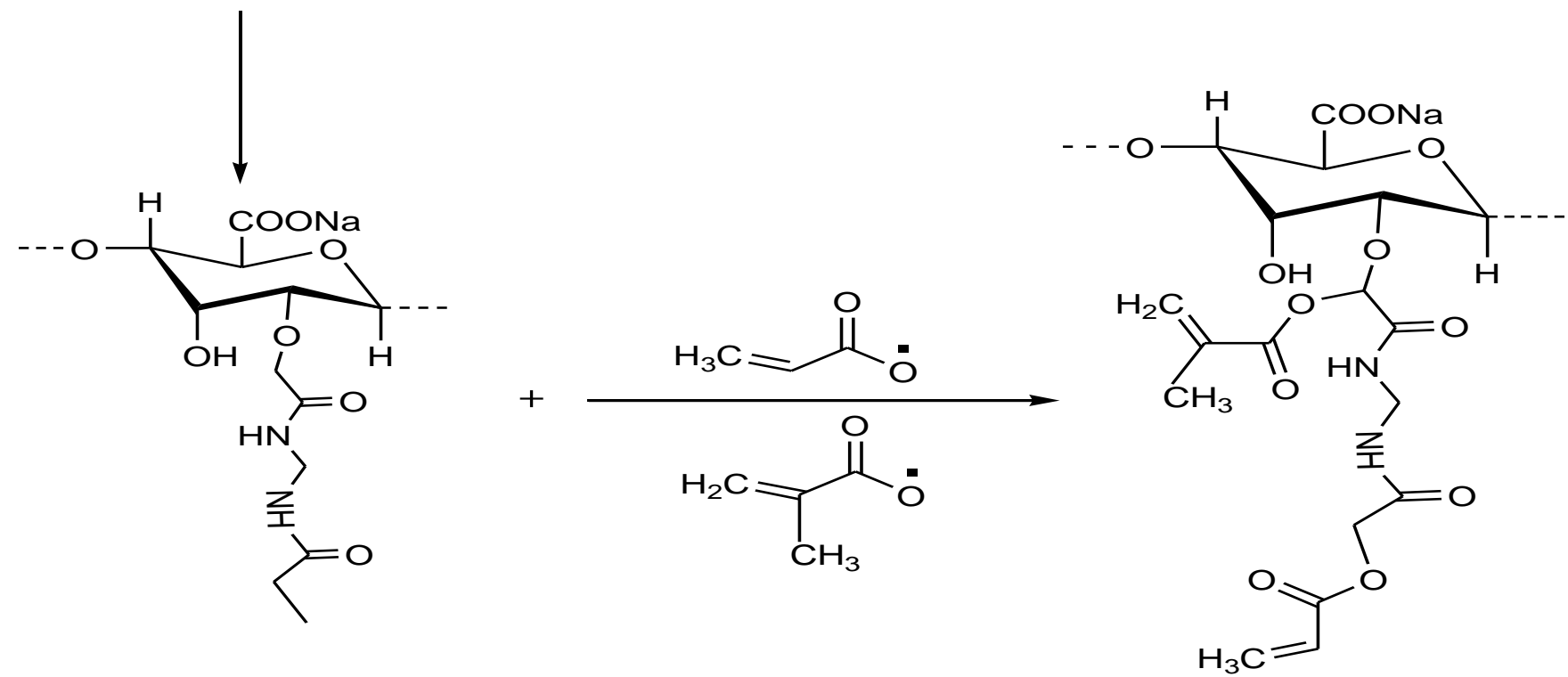

Scheme (1): Possible mechanism reactions for the graft polymerization of Na Alg/AcA/MAcA in presence of MBA 


\subsection{Factors Affecting the Swelling percentage of the Prepared Co-polymer}

The swelling property of the prepared copolymer is one of the most affecting parameters that should be studied to identify its physical and chemical behavior especially the hydrophilic property. The main factors that influence the swelling percentage of the prepared polymer include the following:

\subsubsection{Effect of irradiation dose on swelling percentage}

The effect of different gamma irradiation doses from 5.0 to $30 \mathrm{kGy}$ on the swelling percentage of the prepared grafted copolymer was studied. The result obtained showed that using irradiation doses from 5-10 kGy was found to be not enough for complete polymerization. Accordingly, the effect of irradiation doses from 15-30 kGy on the swelling percentage was studied as shown in Fig. (1).It is clear from this Figure that the swelling $\%$ decreases with increasing the irradiation doses. This is due to the increase in amount (density) of crosslinked network structure at higher irradiation doses [15] which may cause compaction of the copolymer. Therefore, the irradiation dose was fixed at $15 \mathrm{kGy}$ as it is sufficient for the polymerization process.

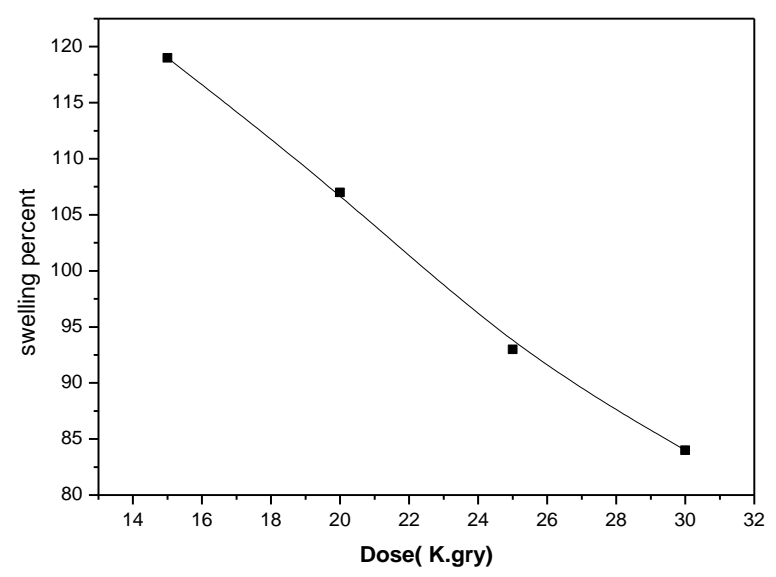

Fig. (1): Influence of the irradiation dose on the swelling percentage

Increasing the gamma radiation, the crosslinking of the matrix increased due to generation of free radicals and the swelling degree decreased. Increasing the gamma radiation and crosslinking at specific limit made the prepared polymer compact or inert material.

\subsubsection{Effect of crosslinker concentration on swelling percentage}

The effect of adding different concentrations of MBA, as a chemical crosslinker, ranged from 0.01 to 0.06 mass $\%$ on the swelling percentage was studied.
This was conducted at a total monomer concentration of 30 mass \% (20\% AcA and 10\% MAcA) and 0.4wt $\%$ $\mathrm{NaAlg}$ with an irradiation dose of $15.0 \mathrm{kGy}$. The results shown in Fig. (2) demonstrated that the swelling percentage decreases with increasing the concentration of the crosslinker. The decrease can be attributed to the higher increase in crosslinking with the polymeric chain which may cause a decrease of diffusivity of water through the network structure and a shrinkage of the polymer may occur[16].Therefore, the concentrations of MBA was fixed at $0.03 \mathrm{wt} \%$ which it is quite sufficient for polymerization without causing polymer shrinkage.

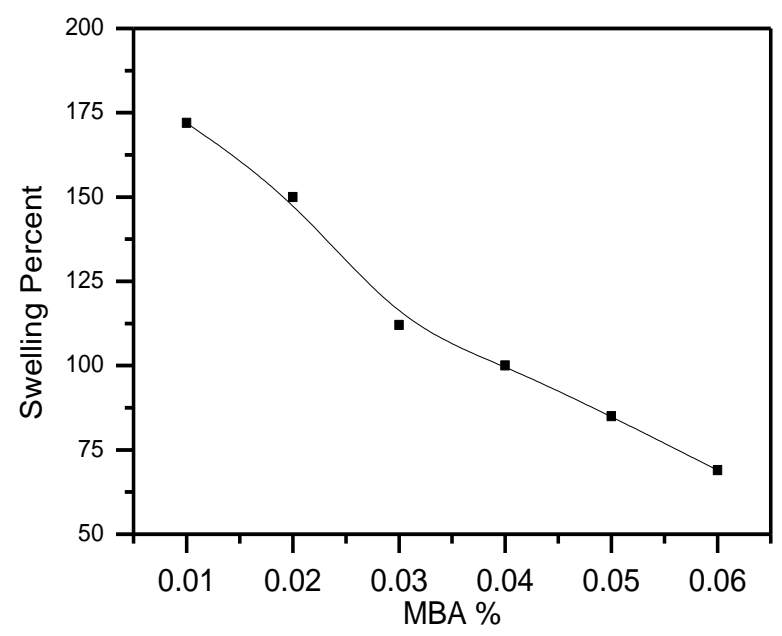

Fig. (2): Influence of the MBA concentration on the swelling percentage

\subsubsection{Effect of sodium alginate on swelling percentage}

Sodium alginate concentration plays an important role in the formation of $(\mathrm{NaAlg} / \mathrm{AcA} / \mathrm{MAcA})$ polymer. The effect of different concentrations of sodium alginate from $0.1-1 \mathrm{wt} \%$ on the swelling percentage of the prepared copolymer is given in Fig. (3). It is observed from this Figure that increasing $\mathrm{NaAlg}$ concentration leads to the increase in water uptake of the copolymer up to $0.2 \mathrm{wt} \%$ of alginate and then it decreases to $1 \%$. It is reported that the introduction of a hydrophilic polymer such as sodium alginate in the gel network of the copolymer increases its water absorption properties[17]. Besides, it also provides electrostatic repulsive force in the network because of its negatively charged carboxylate functional groups $\left(\mathrm{COO}^{-)}\right.$. On the other hand, a further increase of NaAlg above $0.2 \mathrm{wt} \%$ shows a decrease in the swelling percentage which may be due to filling up of the void spaces of the network chains by excess alginate [18-20]. 


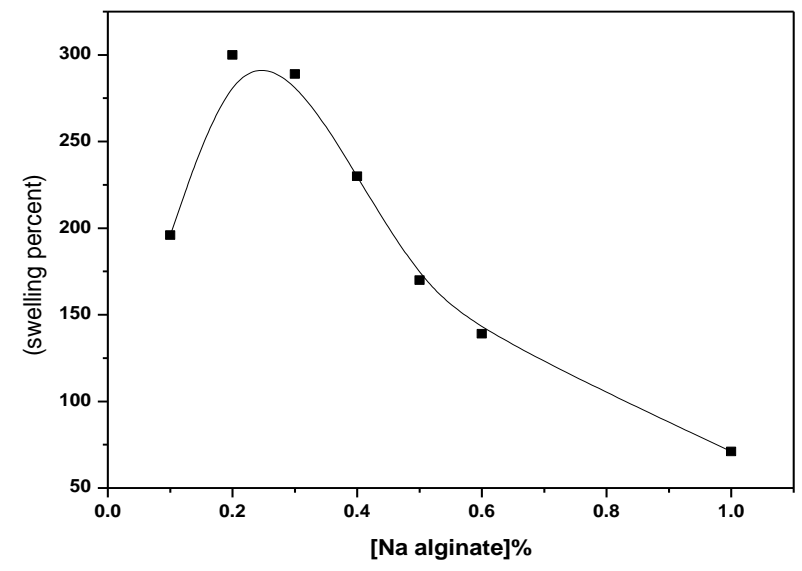

Fig. (3): Influence of the sodium alginate concentration on the swelling percentage

\subsubsection{Effect of pH on swelling Percentage}

The swelling percentage of the prepared copolymer was studied at various $\mathrm{pH}$ values ranged from 1.0 to 13.0. The obtained data revealed that at low $\mathrm{pH}$ values, the swelling percentage increases slightly with an increase in $\mathrm{pH}$ values as shown in Fig. (4). This is because $\mathrm{COO}^{-}$groups of $\mathrm{Na} \mathrm{Alg}$ can be converted to $\mathrm{COOH}$ groups in the acidic solution causing a formation of a hydrogel bonding among - $\mathrm{COOH}$ groups of $\mathrm{Na} \mathrm{Alg}$ and AcA which leads to an increase in the swelling percentage [22-23]. On the other hand, at high $\mathrm{pH}$ values, the carboxylic groups are converted to carboxylate anions with an expansion of the gel network due to electrostatic repulsion which results in a relative increase of swelling percentage [24].

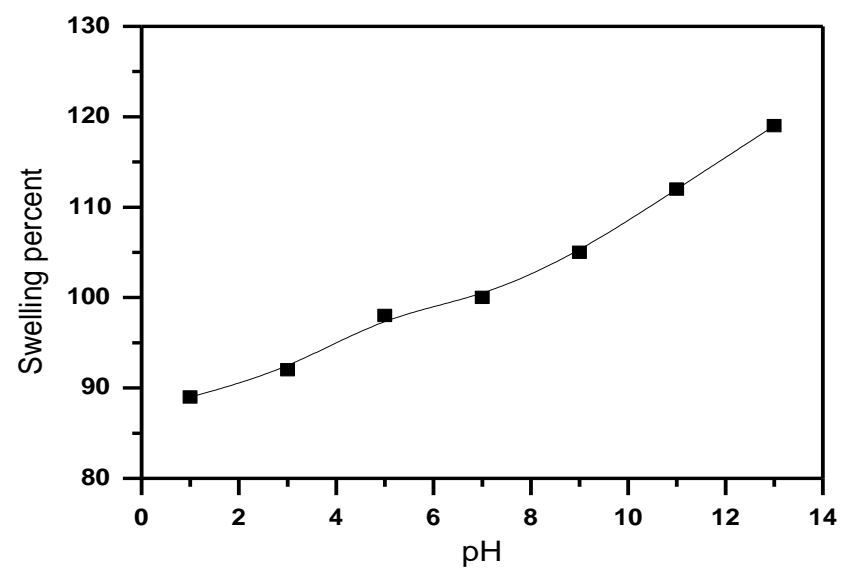

Fig. (4): Effect of $\mathrm{pH}$ on swelling percentage

\subsection{Characterization of the Prepared Co-polymer}

\subsubsection{Scanning Electron Microscope:}

SEM is important to clarify the topography (texture/surface) of a sample, morphology (Size, shape) and approve the compatibility of the monomers. The surface morphology of the prepared grafted copolymer is shown in Fig. (5). The prepared copolymer has a smooth surface and relatively a homogeneous appearance[25]. This indicates an increase in the compatibility between the used constituents.

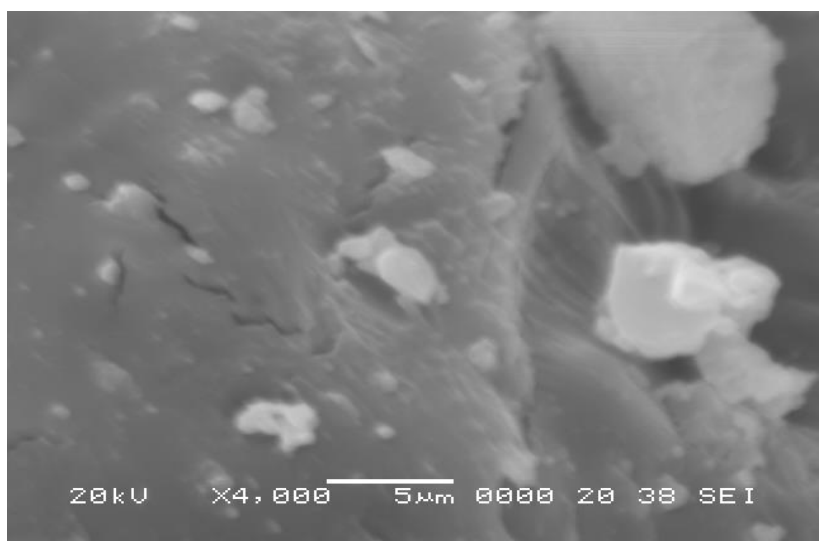

Fig. (5): SEM image of prepared grafted copolymer

\subsubsection{Thermogravimetric analysis}

Fig.(6) shows the thermal decomposition of the prepared grafted polymer. The degradation passes in three stages. The first stage from 50 to $170^{\circ} \mathrm{C}$ which shows that at $170^{\circ} \mathrm{C}$ exhibits a weight loss of $5.819 \%$ with an endothermic peak, which may be due to the removal of absorbed water molecules[26]. The second stage from 170 to $330^{\circ} \mathrm{C}$ with endothermic peaks at $216.57^{\circ} \mathrm{C}$ exhibits a weight loss of $41.465 \%$, which may be due to the release of $\mathrm{COOH}$, water, and $\mathrm{NH}_{3}$ [27]. The third stage from 330 to $600^{\circ} \mathrm{C}$, shows endothermic peaks at $524.69^{\circ} \mathrm{C}$ which exhibits a weight loss of $36.186 \%$, which may be due to the removal of volatile hydrocarbons and complete degradation to the oxide form [28]. The results showed that the polymer possesses thermal stability until near $400^{\circ} \mathrm{C}$.

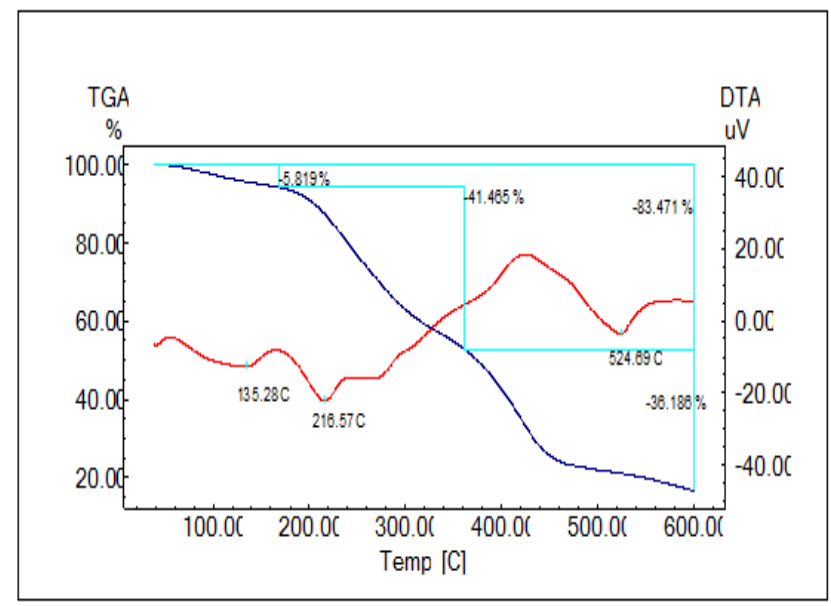

Fig. (6): Thermal analysis of prepared grafted copolymer 


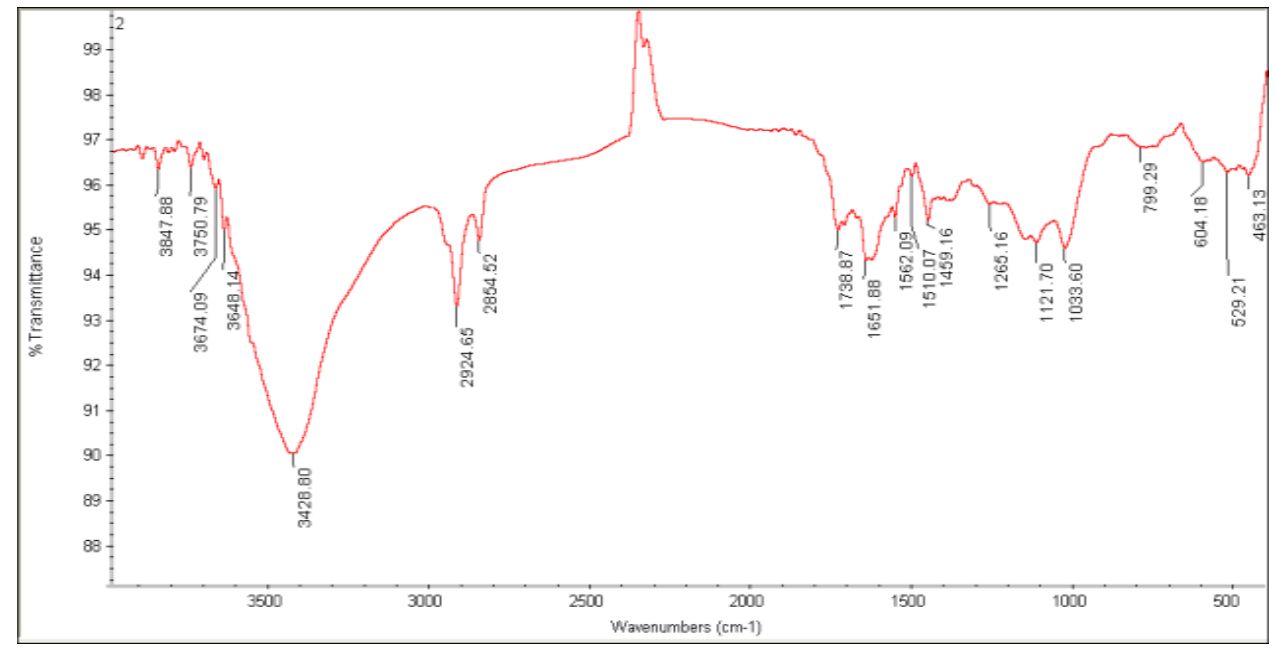

Fig (7): FTIR of the prepared polymer

\subsubsection{FTIR spectra}

FTIR spectroscopy was studied to identify the functional groups of the prepared grafted copolymer (Fig. 7).From literature, the FTIR of sodium alginate showed characteristic absorption bands of hydroxyl, ether and carboxylic functional groups, stretching vibration of $\mathrm{O}-\mathrm{H}$ bands appeared in the range of 3600$3000 \mathrm{~cm}^{-1}$. Stretching vibration of aliphatic C-H was observed at 2920-2850 $\mathrm{cm}^{-1}$ [29] and a stretching band of the carbonyl group $\mathrm{C}=\mathrm{O}$ at $1750 \mathrm{~cm}^{-1}$. Two peaks at 1649 and 1460 were attributed to $\mathrm{COO}$ (asymmetri-c )and $\mathrm{COO}$ (symmetric) groups, respectively. The FTIR spectrum of sodium alginate also shows a strong broad peak between 1107 and $935 \mathrm{~cm}^{-1}$ which is the characteristic peak for polysaccharide structure attributed to $\mathrm{C}-\mathrm{O}$ stretching vibration. A characteristic peak of NaAlg appeared at $840 \mathrm{~cm}^{-1}$ which is attributed (Na-O).

The FTIR spectrum of the prepared copolymer, as observed in Fig.(7), shows bands at 3428, 2924 and 2854 $\mathrm{cm}^{-1}$ which are characteristic for stretching vibration of $\mathrm{O}-\mathrm{H}, \mathrm{C}-\mathrm{H}$, and $\mathrm{CH}_{2}$, respectively. The characteristic stretching vibration bands at 1562 and $1033 \mathrm{~cm}^{-1}$ are attributed to $\mathrm{NH}_{2}$ and alcoholic $\mathrm{OH}$, respectively [30]. The band at $1459 \mathrm{~cm}^{-1}$ is for $\mathrm{C}-\mathrm{H}$ bending of $\mathrm{CH}_{3}$. The intensity of the stretching band of the carbonyl group $\mathrm{C}=\mathrm{O}$ at $1750 \mathrm{~cm}^{-1}$ increased and shifted to $1738 \mathrm{~cm}^{-1}$. The peak of NaAlg which appeared at $840 \mathrm{~cm}^{-1}$ was not obtained in the spectrum of the grafted copolymer. The FTIR spectrum confirmed that the radiation-induced chain degradation of $\mathrm{NaAlg}$ proceeds by the scission of the glycosidic bonds and the formation of unsaturated double bonds without a significant change in chemical structure[31].

\section{CONCLUSION}

The grafted copolymer was prepared by gamma radiation-induced copolymerization of $\mathrm{NaAlg}$ with $\mathrm{AcA}$ and MAcA in the presence of an MBA as a crosslinker. The optimum conditions for preparing the copolymer was fixed at 30 mass $\%$ of total monomers ( $20 \%$ AcA and $10 \% \mathrm{MAcA}$ ) and $0.4 \mathrm{wt} \%$ sodium alginate at an irradiation dose of $15.0 \mathrm{kGy}$ which displays a suitable swelling percentage using $0.03 \%$ of MBA as a crosslinker. The swelling percentage decreases with increasing irradiation dose from 15-30 kGy and MBA crosslinker concentration from 0.01-0.06, while the swelling percentage increases with increasing the $\mathrm{pH}$ solution from 1-13. It also increases with increasing $\mathrm{Na}$ Alg concentration till $0.2 \mathrm{wt} \%$, after which it decreases till $1.0 \mathrm{wt} \%$.

The surface morphology of the prepared grafted copolymer shows a smooth surface and relatively homogeneous appearance. The Thermo-gravimetric analysis of the prepared grafted copolymer shows a good thermal stability suitable for all the domain of the practical application in which this polymer is used till near $400^{\circ} \mathrm{C}$.

\section{REFERENCES}

[1] Ö. Erdem1, Y. Saylan, M Andaç, and A. Denizli.(2018) . Molecularly Imprinted Polymers for Removal of Metal Ions: An Alternative Treatment Method. Biomimetics. 3(38). pp 1-17.

[2] R. P Babu,K. O. Connor, and R. Seeram. (2013) Current progress on bio-based polymers and their future trends. Progress in Biomaterials. 2(1). doi: 10.1186/2194-0517-2-8. 
[3] A.Krishinasailaja, P. Amareshwar, and P.Chakravarty.(2011) Different techniques used for the preparation of nanoparticles using natural polymers and their application. Int J Pharm Pharm Sci. 3( 2), 45-50

[4] I. H. Lone, E. Kowsalya and L. J.Rebecca. (2016)Alginate fiber from brown algae. Der Pharmacia Lettre. 8 (8). 68-71.

[5] A Salisu, A.A. Naim, M.M. Sanagi.(2013). Chemical Modification of Marine Polysaccharide (Alginate) By Free-Radical Graft Copolymerization- a Short Review. IOSR Journal of Applied Chemistry. 4(3). 39-44.

[6] M. N. Mohamad Ibrahim, S. L. Lim, M. R. AhmedHaras, and F. S. Fayyadh. (2014) Preparation and Characterization of Lignin Graft Copolymer as a Filtrate Loss Control Agent for the Hydrocarbon Drilling Industry. BioResources, 9(1). 1472-1487.

[7] V. H. Pino-Ramos, A. Ramos-Ballesteros, F. López-Saucedo, J. E. López-Barriguete, G. H. C. Varca and E. Bucio. (2016) Radiation Grafting for the Functionalization and Development of Smart Polymeric Materials". Topics in Current Chemistry, 374(5). doi:10.1007/s41061-016-0063-X.

[8] T. R. Dargaville, G. A. George, D. J. Hill and A. K. Whittaker (2003) High energy radiation grafting of fluoropolymers. Progress in Polymer Science. 28(9).1355-1376.

[9] Kumar, D., Pandey, J., Raj, V. and Kumar, P. (2017)A Review on the Modification of Polysaccharide Through Graft Copolymerization for Various Potential Applications. The Open Medicinal Chemistry Journal, 11(1). 109-126.

[10] Maykel G. Torres, Manuel G. Pérez, Jorge C. Cortez, Rogelio R. Talavera.(2012). Gamma Radiation-Induced Graft Copolymerization of Typical Monomers onto Poly (3-hydroxybutyrate). International Journal of Science and Advanced Technology (ijsat) 2(9). 106-119.

[11] H.M.H. Gad, Mostafa M. Hamed, H.M.M. Abo Eldahab, M.E.Moustafa, S.A.El-Reefy.(2017) Radiation-induced grafting copolymerization of resin onto the surface of silica extracted from rice husk ash for adsorption of gadolinium. Journal of Molecular Liquids 231. 45-55.

[12] R. Bardajeea, A. Pourjavadib, R. Soleymanb, and N. Sheikh.(2010)Gamma Irradiation Mediated Synthesis of a New Superabsorbent Hydrogel
Network Based on Poly(acrylic acid) Grafted onto Salep. J. Iran. Chem. Soc. 7(3).652-662.

[13] S. Sultana, M. R. Islam, N. C. Dafader and M. E. Haque.(2012) Preparation of carboxymethyl cellulose/acrylamide copolymer hydrogel using gamma radiation and investigation of its swelling behavior.Journal of Bangladesh Chemical Society, 25(2). 132-138.

[14] X. Zhou, H. A. Essawy, M. F. Mohamed, H. S. brahim, N. S. Ammar.(2018) Grafting polymerization of acrylic acid onto chitosan-cellulose hybrid and application of the graft as highly efficient ligand for elimination of water hardness: Adsorption isotherms, kinetic modeling and regeneration. Journal of Environmental Chemical Engineering 6, 21372147

[15] Sokker, H. H., El-Sawy, N. M., Hassan, M. A., \& El-Anadouli, B. E,(2011) Adsorption of crude oil from aqueous solution by hydrogel of chitosan based polyacrylamide prepared by radiationinduced graft polymerization. Journal of Hazardous Material.190(1-3). 359-365.

[16] HV Chavda, CN Patel. (2011) Effect of crosslinker concentration on characteristics of super porous hydrogel. Int J Pharm Investig. 1(1). 17-21.

[17] Maya D.Pinhas, Havazelet B. Peled. (2010)A quantitative analysis of alginate swelling .Carbohydrate Polymers. 79. 1020-1027.

[18] Lee, K. Y., \& Mooney, D. J. (2011) Alginate: Properties and biomedical applications. Progress in Polymer Science.37(1). 106-126.

[19] Işıklan, N., \&Kurşun, F.(2012) Synthesis and characterization of graft copolymer of sodium alginate and poly(itaconic acid) by the redox system. Polymer Bulletin, 70(3). 1065-1084.

[20] Yaoji Tang, Qiang Wang, Bin Zhou, Dong Ma, Ze Ma, and Linhui Zhu. (2015)Synthesis of Sodium Alginate Graft Poly (acrylic acid-co-acrylamide)/ Kaolin Composite Hydrogel and the Study on its Sorption of Rhodamine B. Polymers \& Polymer Composites, 23( 7). 467-474.

[21] Alonso, G. J., Rivera, J. L. A., Mendoza, A. M. M., \& Mendez, M. L. H. (2007). Effect of temperature and $\mathrm{pH}$ on swelling behavior of hydroxyethylcellulose-acrylamide hydrogel.ePolymers. 7(1).epolymer. 1-9 
[22] Xu, X., Bai, B., Ding, C., Wang, H., \&Suo, Y. (2015) Synthesis and Properties of an Eco-friendly Superabsorbent Composite by Grafting the Poly(acrylic acid) onto the Surface of DopamineCoated Sea Buckthorn Branches. Industrial \& Engineering Chemistry Research, 54(13). 32683278 .

[23] Sadeghi, M. and Heidari, B. (2011) Crosslinked Graft Copolymer of Methacrylic Acid and Gelatin as a Novel Hydrogel with $\mathrm{pH}-$ Responsiveness Properties.Materials, 4(3). 543-552.

[24] Sohail, K., Khan, I. U., Shahzad, Y., Hussain, T., and Ranjha, N. M. (2014) pH-sensitive polyvinylpyrrolidone-acrylic acid hydrogels: Impact of material parameters on swelling and drug release. Brazilian Journal of Pharmaceutical Sciences, 50(1).173-184.

[25] M.Sadeghi, A.Godarzi, F.Khani, S.MirdarikVande, H.Sadeghi and H.Shasavari. (2014)Synthesis of a Novel Biopolymer-based alginate Superabsorbent Hydrogel. Bull. Env.Pharmacol.Life Sci. 3 (2). 169174.

[26] Thakur, S., andArotiba, O. (2017)Synthesis, characterization and adsorption studies of an acrylic acid-grafted sodium alginate-based $\mathrm{TiO}_{2}$ hydrogel nanocomposite. Adsorption Science \& Technology, 36(1-2). 458-477.
[27] Kenawy, E.-R., Azaam, M. M., and El-nshar, E. M.(2017) Sodium alginate-g-poly(acrylic-acid-co2-hydroxyethyl methacrylate)/montmorillonite superabsorbent composite: Preparation, swelling investigation, and its application as a slow-release fertilizer. Arabian Journal of Chemistry doi:10.1016/j.arabjc.2017.10.013

[28] S.Huaand A.Wang. (2009)Synthesis, characterization and swelling behaviors of sodium alginate-g-poly (acrylic acid)/sodium humate superabsorbent. Carbohydrate Polymers. 75. 79-84

[29] Campos-Vallette, M. M., Chandía, N. P., Clavijo, E., Leal, D., Matsuhiro, B., Osorio-Román, I. O., and Torres, S. (2010) Characterization of sodium alginate and its block fractions by surface-enhanced Raman spectroscopy. J. Raman Spectrosc. 41. 758763.

[30] Ranjit Mohapatra, Ashok K. Swain, Roomky Mohapatra , Pradeep K Rana, and Prafulla K Sahoo. (2005) Poly(2-Hydroxy Ethyl Methacrylate-coAcrylic Acid) as Novel Biodegradable Macroporous Hydrogel. Polymers \& Polymer Composites, 13( 8). 807-814.

[31] H.L.Abdel-Mohdy.(2017) Radiation-induced degradation of sodium alginate and its plant growth promotion effect. Arabian Journal of Chemistry. 10. 431-438. 